\section{CARDAT: A computer program for the acquisition and analysis of cardiovascular data}

\author{
RICHARD A. GALOSY, SHELLY I. SAFFER, \\ and SHIRLEY J. FOX \\ Departments of Cell Biology, Medical Computing. \\ and the Pauline and Adolph Weinberger Laboratory \\ of Cardiopulmonary Research
}

University of Texas Health Science Center, Dallas, Texas 75235

Because of the vast quantities of cardiovascular data collected in our laboratory, we have designed and implemented a general computer program which allows us to preprocess the major cardiovascular variables of interest to psychophysiologists and cardiovascular physiologists.

Hardware. The general hardware configuration of our system consists of a PDP-12 with 8K core memory, KW12-A programmable clock, RK8E disk, one tape transport, AD-12 analogdigital converter with 8 channels, VR-12 visual display, standard teletype and small line printer. Although our configuration utilizes the RK8E disk pack, another tape transport may be substituted. Output of data can be made to a standard teletype if no line printer is available.

Software. The programs are written in FORTRAN II and SABR (a Digital Equipment Corporation assembly language for $8 \mathrm{~K}$ systems) and run under OS/8 on the PDP-12. The program package is composed of several subroutines to be described below.

In general, the program is designed to accept four analog signals from an FM tape recorder. These signals are then averaged over blocks of time within three separate experimental conditions on each computational run. In addition to A-D conversion and averaging of the four primary signals, several other measures are computed and averaged prior to data output. The experimenter has control over duration of experimental conditions and time blocks to be averaged. If the resulting waveforms suggest that one or more of the input signals may have been influenced by electrical artifacts, the program allows the user to rearrange the data and to display the results on the VR-12 display unit. Artifactual data can then be visualized and corrected through the use of several simple commands to be described below.

Data. The program is currently used to analyze data which is stored on analog FM tape with voice log documentation. Four primary cardiovascular measures-left ventricular pressure (LVP), left atrial pressure (LAP), aortic pressure (AOP), and aortic flow (AOF)-are input from the tape recorder to the AD-12 analog-digital converter of the PDP-12 computer. Using the LVP signal as an input to trigger, the computer program computes the following from the four primary inputs: maximum rate of LVP (LVDP/dt), maximum developed LVP, minimum developed LVP, mean LAP, systolic and diastolic AOP, maximum rate of rise of $A O P(A O D P / d t)$, heart rate (HR), stroke volume, (SV), and total peripheral resistance (TPR).

Calibration procedures. Calibration is done by subroutine CALDAT, which calculates calibration values in array CAL and stores them on DSK in file 'CALFLE.' CALDAT accepts baseline data as a calibration signal. For this reason, clock and trigger must be set prior to calibration. The real time clock is programmed to overflow every $5 \mathrm{msec}$ and LVP is used as input to the Schmitt trigger associated with the clock to signal an external event corresponding to a cardiac cycle. Each input channel may

Development of this program was funded in part by the Harry S. Moss Heart Foundation and NIH Program Project Grant HL06296-14. Richard A. Galosy was a research fellow of the Texas, Dallas Chapter, and American Heart Association. Reprint requests should be addressed to Richard A. Galosy, University of Texas Health Science Center, Department of Cell Biology, 5323 Harry Hines Boulevard, Dallas, Texas 75235. be displayed on the VR-12 by setting of the left switches on the PDP-12 console. The trigger is properly set when a single beat is displayed on the VR-12 at each trigger occurrence.

Prior to calibration of each signal, the signal number and signal expected are printed on the teletype. Sampling begins when any teletype key is depressed with the exception of key ' $\mathrm{N}$ ', which allows the user to skip calibration of the current channel.

CALDAT calibrates Channels 10 through 14 by sampling for $5 \mathrm{sec}$, collecting every other sampled point for display. At the end of $5 \mathrm{sec}$ data collection, 500 points are displayed on the VR-12 at half their original magnitude by subroutine DISML. Analog Channel 3 is sampled by CALDAT (value is in Common) and displayed, by subroutine LINE, as a horizontal line on the VR-12. The line should be set by manipulation of Channel 3 so that it intersects some known low value of the data displayed.

Display is interrupted by teletype keys $S$ and $C$. ' $S$ ' causes a 5 -sec resampling of the same channel, after which the display is resumed. ' $C$ ' causes channel calibration. The real low value is input by the user from the teletype, and the display is resumed. The computer low value is twice the line value, since the display is halfsize. The high value is selected in the same way and read from the teletype. To avoid division by zero when the computer high value equals the computer low value, the channel is resampled. The CAL factor is computed:

$$
\operatorname{CAL}(J J, 2)=\frac{\text { Real High }- \text { Real Low }}{\text { Computer High }- \text { Computer Low }}
$$

At the completion of calibrating the high and low values for the current signal the computer types "channel ok?" A reply of ' 1 ' moves the calibration routine to the next channel. Any other reply allows for recalibration of the current signal. On completion of calibration, the CAL array is written to disk in file 'CALFLE' and printed on teletype. The program then chains to subroutine ADSAM.

Data acquisition. Prior to the start of each data acquisition run, the computer requests the duration of the time block to be averaged. The minimum averaging block is 1 sec; maximum duration is $300 \mathrm{sec}$. After the time duration has been entered, the LVP trigger signal is adjusted, the left console switches are set to view either of the four input channels, and a teletype key is depressed to start sampling by subroutine ADSAM.

Subroutine ADSAM samples Analog Channels 10-14, storing and computing averages for the predetermined averaging time duration. This is continued until a teletype key is depressed signaling a change to the next experimental condition. The acquisition program stops after three experimental conditions have been completed. When the third experimental condition is terminated, data output begins.

Data output. Subroutine DOUT converts double precision values in Common to Real values, applies calibration factors, prints corrected values on the line printer and writes all results to the disk. For each run, a file name is input from teletype by the user which initiates data printout.

The mean values are computed as follows (the sample rate is $.005 \mathrm{sec}$ ):

(1) Mean LAP $=\frac{\text { LAP }-\left(512^{*} \# \text { of sampled points }\right)}{\text { \# of sampled points }}$

$$
=\frac{\text { flow }}{\# \text { triggers }} * \text { sample rate }
$$

$$
\begin{aligned}
= & \frac{\# \mathrm{R}-\mathrm{R} \text { intervals } *(5 \mathrm{msec} \text { intervals } / \mathrm{min})}{\text { total time of } \mathrm{R}-\mathrm{R} \text { intervals }} \\
& \text { Total time of } \mathrm{R}-\mathrm{R} \text { intervals }= \\
& \text { \#amples }{ }^{*} \text { sample rate }
\end{aligned}
$$


(4) $\mathrm{dp} / \mathrm{dt}=\frac{\text { developed pressure }}{\text { sample rate }}$

(5) TPR $=\frac{\overrightarrow{\mathrm{X}} \text { AOP }}{\text { cardiac output }} * 100$ PRU

$\mathrm{PRU}=1 \mathrm{~mm} \mathrm{Hg} / 1 \mathrm{ml} / \mathrm{sec}$

$\overline{\mathrm{X}} \mathrm{AOP}=$ diastolic $\mathrm{AOP}+1 / 3$ (systolic AOP - diastolic AOP)

(6) cardiac output $=S V * H R$

On completion of all output, DOUT may chain to CALDAT for recalibration, or to ADSAM for more data acquisition with the same CAL factors, or to AVIEW for a look at the data, or end data acquisition, chaining to DSKTAP. DSKTAP copies files written by DOUT from disk to link tape.

Viewing of averaged data. After data has been processed through output routine DOUT and data file written to DSK, the user may choose to view the same data by CHAINING to AVIEW, a data acquisition routine which samples and stores running sums of up to six constructive analog channels input and displays the averaged waveform of each channel upon request.

To use AVIEW the investigator enters the duration of the averaging block (this should be the same as that used in the file to be modified) and the number of consecutive channels being sampled. The trigger being displayed in real time on the CRT is adjusted. Any teletype key struck except ' $G$ ' initiates sampling. Sampling begins with the first occurrence of a trigger. Channels are sampled consecutively until completion of preset time interval, or a teletype key is struck, at which time the teletype bell indicates that the tape recorder should be turned off. By setting the left console switches to indicate a channel, one can display the appropriate averaged data points. The display can be positioned on the CRT by manually adjusting an external potentiometer. Displayed points of the waveform can be selected by adjusting another potentiometer (which controls a horizontal line on the CRT), or by positioning a teletype-controlled dot which appears on the waveform. While the display is active, the user may type any of the following teletype keys to obtain the result indicated.

(IDIS refers to the brightened dot on the waveform.)

(The waveform is contained in Array J.)

\section{KEYS}

\section{OPERATIONS}

$>$ Move IDIS one point right. This is used to recompute SV with command $\mathrm{S}$.

$<$ Move IDIS one left. Same as above.

L Sample left switches, average indicated channel. Determines channel to be averaged at this time.

M Take max value (IC) and the relative zero levels of the pressure waveform and find the change in pressure of points (dp) from $J$ (1) to $J$ (IDIS). Save max, min, and $d p$ so the LVP and LVDP/dt and/or AOP and AODP/dt can be computed later.
$X$ Take the value of horizontal line (IC) to represent the mean value of the waveform being displayed. This allows the investigator to visually select an average.

$S$ Calculate the sum, in double precision, of the points displayed. Begin at $J$ (IDIS), which is baseline, stop at next occurrence of baseline. Do not include values above horizontal line. Save sum in J recomputing SV.

Following the above commands, display is resumed.

C Call subroutine OUTDAT. On return, ring bell. Control returns to trigger set-up, above. Saves recomputed data.

R Restart. All previously collected data for this run is lost and programs begin again.

G Chain to OUTADJ without writing any more to disk.

Subroutine OUTADJ will update tha data file. User types ' 1 ' to replace, and enters name of old data file, which is read from DSK. Coordination between current file and update file is by period number. Thus, for a data set, when current equals update period, each value in $\mathbf{J}$ not flagged 'empty' is calibrated and stored in SAV. When current < update period, current data is output, update data is retained pending a period match. When current $>$ update period, current data is retained; update data is discarded. When all $\mathrm{J}$ values have been calibrated and stored, SAV is output to DSK and to the line printer.

Heart rate is recalculated if there has been at least one update to the current data and if the difference between current and update heart rate is greater than 2. TPR is recalculated if TPR, stroke volume, or heart rate has been changed. Calibration values are applied through calls to Function CALB.

When the current data has been exhausted, the user is given the same options (CHAINED) as in DOUT, i.e., recalibrate, sample, view, or end sampling and copy files from disk to tape.

Discussion. The computer program discussed in this paper was designed to allow the user as much flexibility as possible within the limitations of the hardware configuration and data to be analyzed. Toward this goal, CARDAT allows up to three experimental conditions to be sampled in one computer run, i.e. pre- or baseline period, experimental period, and post-period. The duration of each of these time periods may be controlled by the user. In addition, the user has control over the duration of the averaged time block. If one wishes to obtain one value for each experimental condition, he/she would choose an averaging block duration equal to the longest experimental condition. Shorter conditions will be averaged until the user terminates the condition by depressing a teletype key. On the other hand, if the user chooses to obtain, for example, sec $x$ sec averages of the data, he/she would select a 1 -sec averaging time block. Should the experimenter choose to only average one (or two) experimental conditions, they would simply depress the teletype key three times (or two) at the end of the condition.

In conclusion, although the program is currently used for analysis of data store on FM analog tape, it can easily be used in on-line applications by simply connecting the four A-D channels directly to the output of the recording device.

Availability. A program listing, flow charts, and sample output may be obtained upon request from the senior author. 